\title{
Bibliografia de Tesis sobre Literatura Iberoamericana Preparadas en las Universidades de Iberoamérica
}

La bibliografía que sigue ha sido preparada por la Comisión de Coordinación de Investigaciones del Instituto Internacional de Literatura Iberoamericana, para evitar la posible duplicación de trabajo en las muchas investigaciones que se hacen ahora sobre literatura iberoamericana. Como esta fase de la actividad de la Comisión es de origen reciente, no hemos recibido todavía datos de todos los países iberoamericanos. Sin embargo, publicamos ahora los ya recibidos, que son, a nuestro parecer, de un valor muy considerable. Esta primera lista incluye todas las tesis preparadas en las instituciones respectivas desde el año 1935 hasta el presente, y además las tesis de doctorado ahora en preparación. Esperamos publicar todos los años en la Revista Imeroamericana una lista cada vez más completa de las tesis acabadas y en preparación, sobre esta materia, en todas las universidades de Iberoamérica.

$\mathrm{El}$ éxito de tal proyecto se debe especialmente al señor profesor Eduardo Neale Silva, miembro de la Comisión, quien acaba de pasar un año en Iberoamérica disfrutando de una beca Guggenheim. Como resultado de las actividades del profesor Neale Silva, hay en muchos países iberoamericanos miembros del Instituto que tienen la bondad de facilitarnos todos los años los datos que necesitamos. Agradecemos a dichos colegas su bondadosa cooperación.

\section{Tesis de Maestro en Letras ya terminadas}

Altamirano Yerks, Alberto Ignacio. "Influencia de la literatura francesa sobre la literatura mexicana". M. L., Universidad de México, 1935. 
Casalduc de Miranda, Ismael. "José de Jesús Esteves: el poeta". M. L., Universidad de Puerto Rico, 1939.

Díaz, Carmen Rosas. "Félix Matos Bernier: su vida y sus obras". M. L., Universidad de Puerto Rico, 1940.

Garcés y Zamudio, Rafael. "Estudio sobre la obra novelesca del señor licenciado José López Portillo y Rojas". M. L., Universidad de México, 1936.

Ibargüengoitia Llaguno, María. "La poesia de López Velarde". M. L., Universidad de México, 1936.

Mergal, Angél M. "José A. Negrón Sanjurjo: su tiempo, su vida y su obra”. M. L., Universidad de Puerto Rico, 1940.

Rojas de Zúñiga, Clementina. "Estudio monográfico acerca de La Quijotita y su prima". M. L., Universidad de México, 1936.

Rosa-Nieves, Cesáreo. "La poesía en Puerto Rico", M. L., Universidad de Puerto Rico, 1935.

Sánchez Palomo Villalobos, María Natalia. "Manuel Gutiérrez Nájera y el cuento". M. L., Universidad de México, 1938.

Silva, Margarita. "El jibaro en la literatura puertorriqueña". M. L., Universidad de Puerto Rico, 1936.

Vargas Cepeda, Gracia María. "El cuento y la novela corta en México, en algunos escritores del siglo XIx". M. L., Universidad de México, 1937.

Zúñiga Rojas, Maria Teresa. "D. Guillermo Prieto: su vida y su obra”. M. L., Universidad de México, 1937.

\section{Tesis de Doctor en Letras y de Doctor en Filosofia y Letras ya terminadas}

Altamirano Yerks, Alberto Ignacio. "Lo maravilloso en el cuento y la novela". Dr. L., Universidad de México, 1937.

Cipriani, Graciela. "Exposición y crítica de las doctrinas de Alberdi". Dr. F. y L., Universidad de Buenos Aires, 1938.

Cometta Manzoni, Aída. "El indio en la poesía de la América española". Dr. F. y L., Universidad de Buenos Aires, 1939.

Corti, Dora. "Florencio Sánchez". Dr. F. y L., Universidad de Buenos Aires, 1936.

Figueroa, Esperanza. "Revisión de Julián del Casal". Dr. F. y L., Universidad de La Habana, 1940. 
Gómez Roca, Clara. "Poesía lírica de Amado Nervo". Dr. F. y L., Universidad de La Habana, 1938.

Gutiérrez López, Antonio. "Nuevas cuestiones sobre el castellano en México". Dr. L., Universidad de México, 1935.

Le Moyne Benge, Frances. "El bardo de Nayarit (última ofrenda)". Dr. L., Universidad de México, 1935.

Moré, Gerardo. "Heredia, cantor de la religión". Dr. F. y L., Universidad de La Habana, 1939.

Oursler, Anna Lovina. "El drama mexicano desde la revolución hasta el año de 1940". Dr. L., Universidad de México, 1940.

Oviedo Villegas, Jesús J. "La literatura gauchesca, dentro de la literatura argentina". Dr. L., Universidad de México, 1935.

Taylor, Bárbara Howland de, "La tradición y la leyenda en la literatura mexicana". Dr. L., Universidad de México, 1936.

Treviño, Alberto. "Armando Godoy". Dr. F. y L., Universidad de La Habana, 1939.

Zamudio Silva, Jorge. "Juan Manuel Fernández de Agüero". Dr. F. y L., Universidad de Buenos Aires, 1939.

\section{Tesis de Doctor en Filosofía y Letras, en preparación}

Brizuela, Dulce María. "José Maria Heredia, poeta amoroso". Universidad de La Habana.

Cartaya, Rosa. "Milanés y su obra literaria". Universidad de La Habana.

Cepero, Elodia. "Anselmo Suárez y Romero y su obra literaria". Universidad de La Habana.

Cortina de Varona, Héctor. "La obra literaria de Ricardo Palma." Universidad de La Habana.

Díaz, Adelaida Clemente. "El teatro de la Avellaneda." Universidad de La Habana.

Gomiz Valdés, Amada. "La novela social cubana durante el primer cuarto del siglo $\mathrm{xx}$ ". Universidad de La Habana.

Hernández Travieso, Antonio. "La filosofía de Félix Varela". Universidad de La Habana.

Hevia, Esther. "La crítica de Enrique Piñeyro". Universidad de La Habana. 
Llerena, Mario. "La cubanidad de José Antonio Saco". Universidad de La Habana.

Malo Rendón, Rina. "Novelas y leyendas de la Avellaneda". Universidad de $\mathrm{La}$ Habana.

Martí Rico, Dolores. "Tres mujeres de América: Alfonsina Storni, Juana de Ibarbourou, Gabriela Mistral". Universidad, de La Habana.

E. K. MAPES,

Universidad de Iowa. 